\title{
Numerical expressions in time domain for electromagnetic fields due to lightning channels.
}

\begin{abstract}
In this paper, by considering the factors that influence the charge height variation along the lightning channel and using Maxwell's equations, general expressions in time domain for the evaluation of electromagnetic fields at an observation point are proposed. The lightning channel is assumed to be perpendicular to the ground surface and the ground conductivity is considered to be perfect. Our results showed that the proposed method has good agreement with the measured values, as we validated the outcome using fields measured at close and intermediate distances from the lightning channel. A set of general equations, which has the ability of evaluating any given channel base current function and current model in time domain is also derived.
\end{abstract}

Keyword: Electromagnetic fields; Lightning; Lightning channel; Return stroke current. 